\title{
A Structure Landmark-Based Radio Signal Mapping Approach for Sustainable Indoor Localization
}

\author{
Tao Liu ${ }^{1,2}$, Xing Zhang ${ }^{3,4,5, *}$, Huan Zhang ${ }^{2}$, Nadeem Tahir ${ }^{2}$ and Zhixiang Fang ${ }^{6}$ (D) \\ 1 College of Resources and Environment, Henan University of Economics and Law, Zhengzhou 450002, China; \\ liutao@huel.edu.cn \\ 2 Key Laboratory of New Materials and Facilities for Rural Renewable Energy (MOA of China), \\ Henan Agricultural University, Zhengzhou 450002, China; zhanghuan5754@163.com (H.Z.); \\ nadeemtahir@163.com (N.T.) \\ 3 Guangdong Key Laboratory of Urban Informatics, the School of Architecture and Urban Planning, \\ Shenzhen University, Shenzhen 518060, China \\ 4 Shenzhen Key Laboratory of Spatial Information Smart Sensing and Services, the School of Architecture and \\ Urban Planning, Shenzhen University, Shenzhen 518060, China \\ 5 MNR Key Laboratory for Geo-Environmental Monitoring of Great Bay Area, the School of Architecture and \\ Urban Planning, Shenzhen University, Shenzhen 518060, China \\ 6 State Key Laboratory of Information Engineering in Surveying, Mapping, and Remote Sensing, \\ Wuhan University, Wuhan 430072, China; zxfang@whu.edu.cn \\ * Correspondence: xzhang@szu.edu.cn
}

check for updates

Citation: Liu, T.; Zhang, X.; Zhang H.; Tahir, N.; Fang, Z. A Structure Landmark-Based Radio Signal Mapping Approach for Sustainable Indoor Localization. Sustainability 2021, 13, 1183. https://doi.org/ $10.3390 /$ su13031183

Academic Editor: James (Jong Hyuk) Park

Received: 15 November 2020

Accepted: 20 January 2021

Published: 23 January 2021

Publisher's Note: MDPI stays neutral with regard to jurisdictional claims in published maps and institutional affiliations.

Copyright: (c) 2021 by the authors. Licensee MDPI, Basel, Switzerland. This article is an open access article distributed under the terms and conditions of the Creative Commons Attribution (CC BY) license (https:// creativecommons.org/licenses/by/ $4.0 /)$
Abstract: Low cost and high reproducible is a key issue for sustainable location-based services. Currently, Wi-Fi fingerprinting based indoor positioning technology has been widely used in various applications due to the advantage of existing wireless network infrastructures and high positioning accuracy. However, the collection and construction of signal radio map (a basis for Wi-Fi fingerprinting-based localization) is a labor-intensive and time-cost work, which limit their practical and sustainable use. In this study, an indoor signal mapping approach is proposed, which extracts fingerprints from unknown signal mapping routes to construct the radio map. This approach employs special indoor spatial structures (termed as structure landmarks) to estimate the location of fingerprints extracted from mapping routes. A learning-based classification model is designed to recognize the structure landmarks along a mapping route based on visual and inertial data. A landmark-based map matching algorithm is also developed to attach the recognized landmarks to a map and to recover the location of the mapping route without knowing its initial location. Experiment results showed that the accuracy of landmark recognition model is higher than $90 \%$. The average matching accuracy and location error of signal mapping routes is $96 \%$ and $1.2 \mathrm{~m}$, respectively. By using the constructed signal radio map, the indoor localization error of two algorithms can reach an accuracy of $1.6 \mathrm{~m}$.

Keywords: indoor localization; fingerprinting; structure landmark

\section{Introduction}

Location information is a necessary component of the Future Sustainability Computing (FSC) framework which integrates diverse policies, procedures, programs and provides amount of potential applications such as mobile computing, robots and pedestrian navigation, augmented reality and other Location Based Service (LBS) [1]. For example, a RSSI-based distance estimation framework [2] was constructed to accurately estimate the location of users, in sustainable indoor computing environment. An infrared human posture recognition method [3] was also developed for intelligent monitoring in sustainable smart homes. Indoor localization and monitoring are important to sustainability technologies and systems. By obtaining the location information, more services and application can be developed to support FSC. 
While outdoor positioning problem can be well solved by using the Global Navigation Satellite System (GNSS), indoor positioning remains a challenge due to the lack of reliable and low-cost indoor positioning technique. There are a number of solutions developed for indoor positioning such as Wi-Fi [4], magnetic [5], UltraWide Band (UWB) [6], image [7], inertial sensor [8], etc. Compare with other indoor localization techniques, Wi-Fi fingerprinting has been widely used in various indoor environments, since it can be easily deployed using existing off-the-shelf mobile devices and wireless network (e.g., 802.11 Wi-Fi infrastructures) without participating of other devices and infrastructures.

Based on routing algorithms [9,10], Wi-Fi signals can achieve relative high throughput, low latency, and high reliability from source to destination in wireless networks. However, the growing demand and diverse traffic pattern of smart devices increase the strain on wireless networks. Some studies attempt to provide solutions to fulfill the requirements of distributed communications. For example, Wang [11] presented a coexistent routing and flooding network, which could conduct routing within the Wi-Fi network and flooding among ZigBee node using a single stream of Wi-Fi packets. Kemal [12] proposed the DeepWiFi protocol, which hardens the baseline Wi-Fi with deep learning and sustains high throughput by mitigating out-of-network interference. It supports embedded platform and provide major throughput gains especially when channels are likely to be jammed. Gilani [13] proposed a Software Defined Networking (SDN) based routing architecture for Wireless Mesh Networks (WMNs). Although these state-of-art Wi-Fi routing algorithms can improve the capacity of communication of wireless network, they cannot directly provide accurate location information of mobile devices for indoor and outdoor locationbased applications.

The principle of Wi-Fi fingerprinting is to calculate location by matching the current WiFi received signal strength (RSS) measurements with a set of RSS measurements with known locations. It is generally based on two phases: offline training phase and online localization phase. In offline phase, the geo-tagged RSS measurements are collected from multiple Wi-Fi access points (AP) and treated as a fingerprint. A number of the collected fingerprints which covers an indoor space is used to construct a radio map. Whereas, in online phase, the location of user is computed by matching the current fingerprint measurement with a constructed radio map. The localization accuracy of Wi-Fi fingerprinting is significantly affected by the quality of radio map. The location accuracy and reliability can be improved when the fingerprints are accurately geo-tagged with high spatial density. However, it also requires more labor and time cost for fingerprint measurement and geo-tagging. For Wi-Fi fingerprinting, the collecting and constructing of radio map is a key issue that limits its large-scale application.

Recently, much effort has been devoted to reducing the intensive cost of manpower and time for radio signal mapping (i.e., collecting and generating radio map). Some researchers have tried to replace the site survey process by employing wireless radio propagation models $[14,15]$. However, due to the unpredictability of signal propagation, signal propagation models do not perform properly in complex indoor environments and lack the potential of their applications. Some researchers [16-19] have focused on development of zero-configuration indoor localization systems which do not require an explicit site survey phase. Instead, they implement a training phase during the use of the systems. For example, Sorour [16] exploits the inherent spatial correlation of RSS measurements to reduce the required calibration of fingerprints and performs a direct localization without a full radio map. Redpin [17] is an adaptive zero-configuration indoor localization system which constructs a fingerprint database by encouraging users to upload self-identified locations. However, Redpin needs a large amount of collaboration data and cannot be applied in the initial stage. UnLoc [18] is a localization scheme which can sense and identify indoor landmarks, e.g., elevators or corridor corners, and uses them to recalibrate the locations, without the need for a site survey. Zee [19] is a localization system that does not need an explicit site survey or calibration. It requires the users to roam in the indoor area to collect information before being able to localize themselves. 
In general, although these systems can be directly applied without a full site surveying process, they require a long training and recalibration process and cannot provide reliable localization results before the initialization and training phase are finished. As a widely used artificial intelligence technique, deep learning has been employed by many indoor localization approaches. For example, Hsieh [20] presented a deep learning-based approach by utilizing transmission channel quality metrics. This approach partitioned indoor area into two-dimensional blocks and train four deep neural networks to estimate the location of RSS and CSI (channel state information). Koike [21] used spatial beam signal-to-noise ratios (SNRs) as mid-grained intermediate-level channel measurement and constructed fingerprinting database by using a deep learning approach. These works have not considered the labor cost of constructing and updating fingerprint database. WiDeep in [22] is a deep learning-based indoor localization system, which combines a stacked denoising autoencoders and a probabilistic framework to handle the noise and instability of Wi-Fi signals. Zahra [23] employed extreme learning machines and the high-level features extracted from autoencoder to improve localization performance in both feature extraction and classification. Wang [24] proposed a stacked denoising autoencoder based feature extraction method to obtain time-independent Wi-Fi fingerprint. A multi-layer perceptron method was used to build a regression model which associated the extracted feature to the corresponding location. These methods can reduce the labor cost required for fingerprinting but also require a relative long time for data training.

Crowdsourcing is an efficient way to reduce the labor and time cost that needed for radio signal mapping. Many studies [25-28] have used crowdsourcing trajectories to construct radio map. For example, WILL [25] investigates unexploited RF signal characteristics and leverage user motions to construct radio map. LIFS [26] applies human motions to connect previously independent radio fingerprints. Recently, AiFiMatch [27] and RCILS [28] take advantage of activity detection and map matching techniques to estimate crowdsourcing trajectory. Later, fingerprints can be calibrated from the trajectory sequence. However, although the accuracy of activity detection method is high, it is difficult to apply these systems in practical use. This could be due to the fact as it assumes that all the activities (e.g., a turn) performed at special locations (e.g., corners or elevators). However, this assumption is vulnerable to the randomness of human activity. If a turning activity happens at corridor open space or room, it will be wrong to match the current location to the special locations recorded in an indoor map.

Landmark is always used as the spatial reference due to its with salient visual and semantic characteristics. It can provide accurate location information and can be used to reduce positioning error of indoor or outdoor positioning systems. During the recent decades, a large amount of studies has focused on developing landmark recognition methods or systems. For example, Cao [29] proposed a landmark recognition algorithm with sparse representation classification and extreme learning machine. The spatial pyramid kernel-based bag-of-words histogram approach was used to extract image feature, and the artificial neural network was trained with extreme learning machine combine with sparse representation classifier for landmark image recognition. Cunha [30] has proposed the Patch PlaNet which considered landmark recognition as a classification problem and extended the PlaNet deep neural network model to perform the classification. Compared with original network, the performance of Patch PlaNet improves the accuracy of landmark recognition by $5-11$ percentages. These works can achieve a satisfactory landmark recognition result. Federico [31] considered the computational time problem in large-scale landmark recognition. The algorithm can drastically reduce the query time associate with outperform accuracy, which can be applied to diverse embedding techniques. However, this algorithm is not suitable for indoor environment with insufficient texture features. Chua [32] proposed a landmark-based indoor navigation approach, which employed a 360-degree camera to recognize landmarks and provide a shortest navigation path using Dijkstra algorithm. Except for image landmark, special sensor data or Wi-Fi signal can also be used as landmark. Sun [33] used both RSS crests and troughs to define a landmark. 
A RSS waveforms smoothing method was implemented on the clustered crowdsourced trajectories, which improved the location accuracy of Wi-Fi landmarks about $24.2 \%$. The change of indoor environment layout and moving pedestrian may decrease the accuracy of Wi-Fi landmarks.

This study intends to utilize spatial structural features, such as intersections or corners, to correct the cumulative error of crowdsourcing trajectories for radio signal mapping. Compared with human activity, spatial structures are visually sensible features existing in physical space. It makes spatial structures, termed as structure landmarks which are more reliable and robust as compare to activity landmarks. Through detecting and recognizing the structure landmarks (e.g., L-junction, T-junction) along a route, the location of the route can be spatially estimated and calibrated. The sampling points from the route can be used to generate fingerprints and construct radio map for indoor localization.

This study proposes a structure landmark-based radio signal mapping method which is sustainable for indoor localization. For an indoor environment, structure landmarks can be easily extracted from a floorplan and stored in a landmark dataset along with their geo-tags. During a radio signal mapping process, multi-sensor data can be collected through smartphone, including video frames, inertial readings and Wi-Fi signals. Based on proposed structure landmark recognition method, video frames and inertial readings can be integrated to recognize the type of structure landmark along a mapping route. The inertial data can also be utilized to roughly calculate the relative location of the route. By matching the recognized structure landmarks with the ones in landmark dataset, the location of the recognized landmarks can be estimated. After that, the recognized landmarks can be used to accurately estimate the location of the mapping route. Finally, a Wi-Fi radio map can be constructed to extract further Wi-Fi fingerprints from a spatially estimated mapping route. An indoor localization experiment is also conducted to evaluate the quality of the constructed radio map.

This paper is organized as follows: Section 2 presents the methodology of structure landmark based indoor radio signal mapping method. Experimental results and comparisons are described in Section 3. Conclusions are drawn in Section 4.

\section{Methods}

The overview of this approach is described in Figure 1. The inputs of this approach are video frames, inertial data and Wi-Fi RSS values, which can be collected by smartphone sensors. Landmark dataset, including type and location attributes of structure landmarks, can be extracted from indoor floor plan. The output of this method is a generated Wi-Fi radio map, which can be indirectly used for indoor localization.

This method firstly defined the type of typical structure landmarks in indoor space. Then, a learning-based classification model was designed for distinguishing the type of structure landmark based on collected inertial sensor data and video frames. Inertial sensor data including accelerator, gyroscope and orientation readings were used to recover trajectory geometry by using the PDR method. Furthermore, heading angle change calculated from gyroscope data was used as a feature to recognize the type of structure landmark. As initial location of trajectory was unknown, a Hidden Markov model (HMM) based map matching method was also developed to match the recognized structure landmarks along the trajectory to a landmark database. The location of identified structure landmarks can be determined, which can be further used to estimate the spatial location of the trajectory. Finally, the sampling points from the trajectory were used to generate a radio map for indoor localization. 


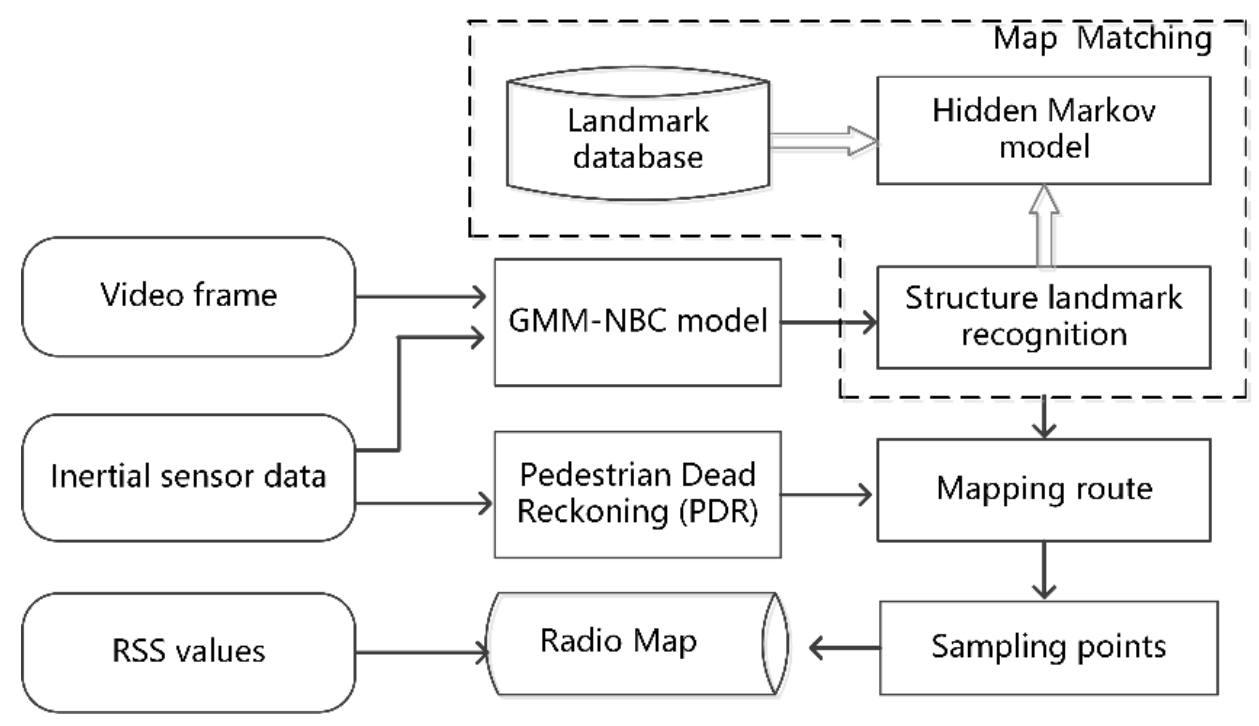

Figure 1. Architecture of the indoor signal mapping system.

\subsection{Types of Structure Landmark}

Structure landmarks are defined as visually salient structures in indoor space that anchors special locations, such as intersections, entrance, corners, etc. As shown in Figure 2, 8 different types of structure landmark have been defined, including FT (T-junction at front angle), LT (T-junction at left angle), RT (T-junction at right angle), LL (L-junction at left angle), RL (L-junction at right angle), EC (end of corridor), CW (corridor to wide area) and WC (wide area to corridor). Each type of structure landmark has a special structural and visual characteristic, which is a basis for landmark recognition. According to the definition, structure landmark dataset can be easily extracted from a floorplan of an in-door space.
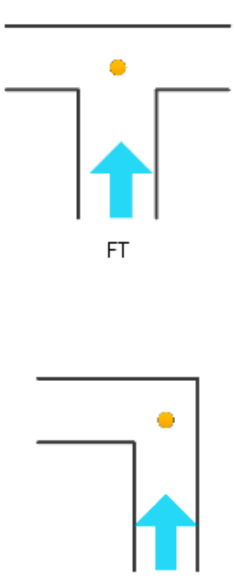

$\mathrm{LL}$

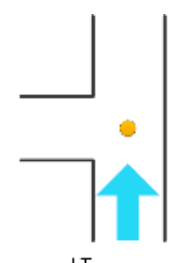

LT

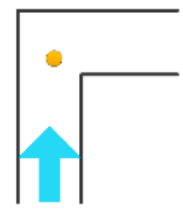

$\mathrm{RL}$

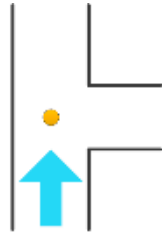

RT

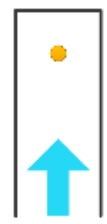

EC
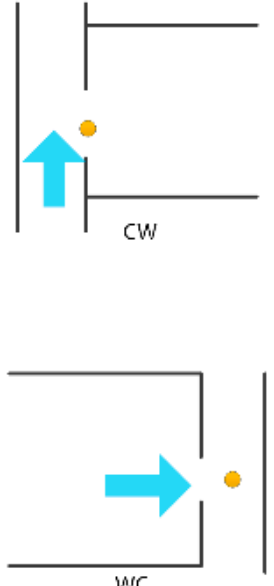

Figure 2. Types of defined structure landmark.

This study intends to integrate both visual and inertial features to recognize the type of a structure landmark. The reason for including visual features in landmark recognition is that visual information has a more reliable relationship with the physical environment as compare to inertial information. This method does not depend on the assumption that all walking activities (e.g., a turning action) happen at special locations (e.g., an intersection). Instead, it employs visual information to sense and detect whether a person is at a special location (i.e., structure landmark). A Gaussian Mixture Model based Naïve Bayesian Classifier (GMM-NBC) is proposed to recognize the type of landmark. 


\subsection{Structure Landmark Recognition}

\subsubsection{Feature Calculation}

One important visual feature for landmark recognition is vanishing segments, which is the line segments toward the vanishing point of a video frame. Vanishing points and vanishing segments are important visual features for various computer vision studies, including scene understanding [34], 3D reconstruction [35], and so on. Indoor scene can be abstracted as three cluster lines with orthogonal direction towards vanishing point. As shown in Figure 3, the vanishing segments towards horizontal (red line), vertical (green line) and infinity direction (blue line) can be extracted from a video frame by using the proposed method in [36]. Using vanishing segments, a visual feature variable is defined as follows:

$$
v_{s}(i)=\frac{\operatorname{sum}\left(L_{h}\right)}{\operatorname{sum}\left(L_{h}\right)+\operatorname{sum}\left(L_{f}\right)}
$$

where $v_{S}(i)$ is the defined feature value of frame $i, L_{h}$ is the vanishing segments towards the horizontal direction, $L_{f}$ is the vanishing segments towards the infinity direction, sum is a function to calculate the total length of line segments. The value of $v_{s}(i)$ reflects the structure characteristics of a scene.

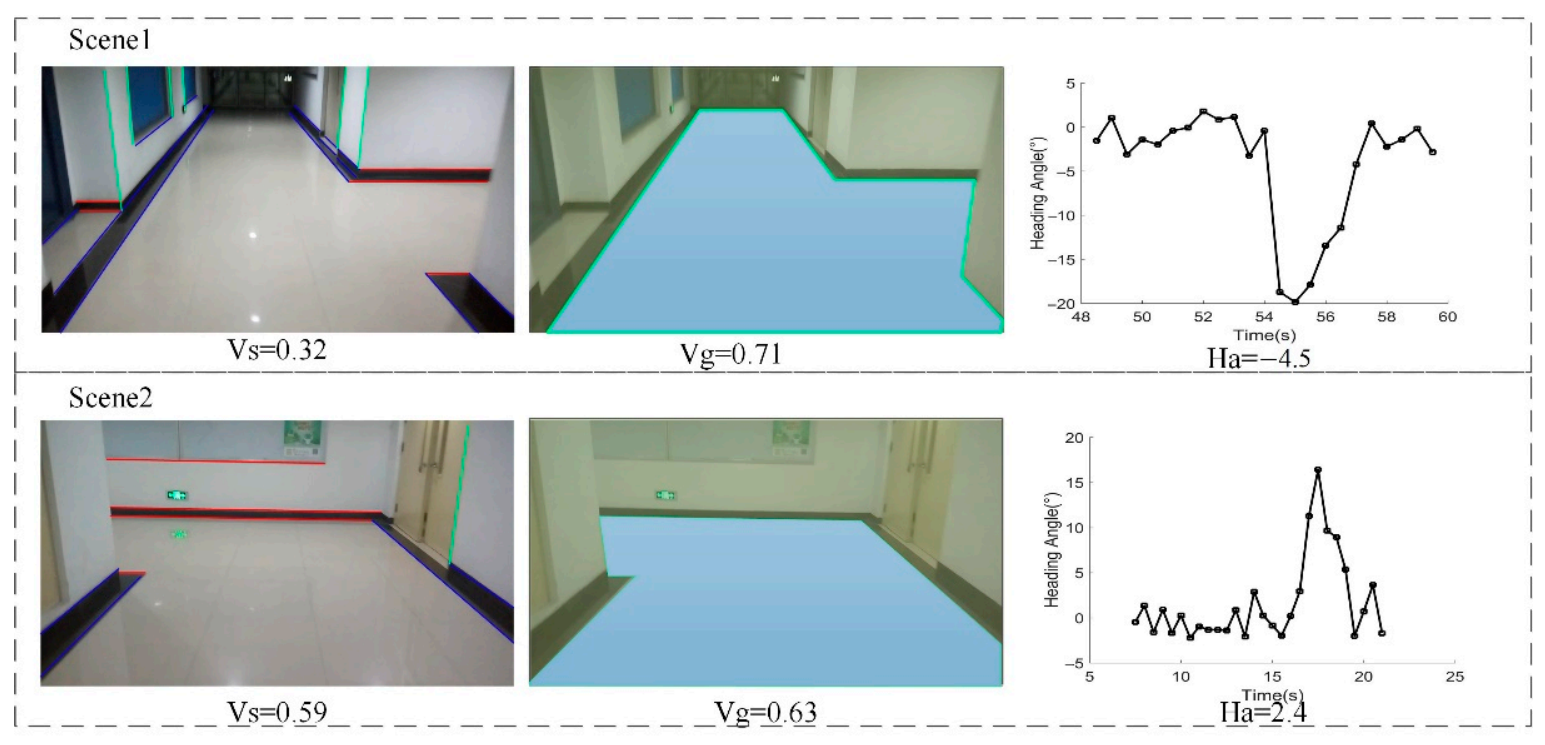

Figure 3. Example of feature values of two different indoor scene.

Another important visual feature is the floor plane of an indoor scene. Floor plane detection is a basic issue for many study fields, such as mobile robotics, navigation and 3D modeling. This method calculates the wall-floor boundary and evaluate the likelihood of horizontal intensity edge line segments by using the algorithm proposed in [37]. The visual feature variable is defined as follows:

$$
v_{g}(i)=\frac{S_{g}(i)}{S(i)}
$$

where $S_{g}(i)$ is the area of an extracted floor plane from frame $i, S(i)$ is the size of frame $i$. The variable $v_{g}$ shows the ratio of floor plane area in an image.

The third feature is heading angle change, which is calculated by using gyroscope readings. When a person walks across a structure landmark, he or she may turn left, turn right or turn around. Different type of structure landmark leads to different walking direction. For example, LL and RL landmark refer to one walk direction, while FT landmark provides two potential walking directions. At the location of EC landmark, a person may 
turn around. Therefore, this method calculates change in heading angle variable using inertial reading. The feature of heading angle change can be calculated as follows:

$$
h_{a}(\mathrm{i})=\sum_{T_{s i}}^{T_{e i}} \int g y r \cdot d t
$$

where $h_{a}(\mathrm{i})$ is the heading angle change of frame $i, T_{s i}$ and $T_{e i}$ are the start and end moments of frame $i$, gyr is the gyroscope readings from smartphone.

For each type of structure landmark, the calculated visual and inertial features are used to construct a feature vector, which can be represent as:

$$
\left\{\begin{array}{l}
\left.V_{s}=\left\{v_{s}(1), v_{s}(2)\right\} \ldots v_{s}(j)\right\} \\
\left.V_{g}=\left\{v_{g}(1), v_{g}(2)\right\} \ldots v_{g}(j)\right\} \\
H_{a}=\left\{h_{a}(1), h_{a}(2) \ldots h_{a}(j)\right\}
\end{array}\right.
$$

where $\left\{v_{s}(j), v_{g}(j) \ldots h_{a}(\mathrm{j})\right\}$ represent the three different feature value of frame $j$. Figure 3 shows an example of feature values for two different indoor scenes. Scene 1 is a RT landmark and Scene 2 is a LL landmark. The value of $v_{s}$ and $h_{a}$ of two scenes are significantly different.

\subsubsection{GMM-NBC Construction}

In this paper, a GMM-NBC model is designed to distinguish the type of structure landmarks. The principle of GMM-NBC is to estimate the joint probability of continuous feature vectors based on parametric probability density function. The highest value of joint probability determines the type of a structure landmark.

Because the distribution of feature value is unknown, this method uses the GMM model to approximate the distribution. GMM is a mixture model with a weighted sum of K component Gaussian densities, which can be used to fit any type of distribution in theory. The GMM can be formulated as:

$$
p(x \mid \lambda)=\sum_{i=1}^{M} w_{i} g\left(x \mid \mu_{i}, \sum_{i}\right)
$$

where $\mathrm{x}$ is a $\mathrm{D}$-dimensional continuous-valued data vector, $\lambda$ is a parameter set which can be defined as: $\lambda=\left\{w_{i}, \mu_{i}, \sum_{i}\right\}$ ( $\left.\mathrm{i}=1,2, \ldots \mathrm{M}\right), w_{i}$ represents the mixture weight which satisfy the constraint as:

$$
\sum_{i=1}^{M} w_{i}=1
$$

$g\left(x \mid \mu_{i}, \sum_{i}\right)$ is the component Gaussian densities function with the mean vector $\mu_{i}$ and the covariance matrix $\sum_{i}$, which can be formulated as follows:

$$
g\left(x \mid \mu_{i}, \sum i\right)=\frac{1}{(2 \pi)^{\frac{D}{2}}\left|\sum i\right|^{\frac{1}{2}}} \exp \left\{-\frac{1}{2}\left(x-\mu_{i}\right)^{\prime} \sum_{i}^{-1}\left(x-\mu_{i}\right)\right\}
$$

In order to use the complete GMM to fit the feature distribution, the parameter set $\lambda$ should be learned on the training phase. There are several techniques available to estimate the parameters of a GMM e.g., moment estimation, minimum $x^{2}$ estimation, Bayesian estimation and maximum likelihood estimation [38]. In this study, we use the maximum likelihood estimation (MLE) to find the model parameters which maximizes the likelihood of GMM given the training data. For sequence of training vectors $X=\left\{x_{1}, x_{2}, \ldots x_{T}\right\}$, the likelihood function of $\lambda$ can be represented as follows:

$$
p(X \mid \lambda)=\prod_{t=1}^{T} p\left(x_{t} \mid \lambda\right)
$$

Because Equation (8) is a non-linear function of parameters $\lambda$ and direct maximization is not possible. This method uses an expectation-maximization (EM) algorithm to solve this problem. Given an initial model $\lambda$, the basic idea of EM algorithm is to estimate a new 
mode $\bar{\lambda}$, such that $p(X \mid \bar{\lambda}) \geq p(X \mid \lambda)$. The new model can then be implemented as an initial model for the next iteration. This process is repeated until some convergence threshold is reached.

On each EM iteration, the estimate of parameters can be represented as follows:

$$
\begin{gathered}
\overline{w_{i}}=\frac{1}{T} \sum_{t=1}^{T} p\left(i \mid x_{t}, \lambda\right) \\
\overline{\mu_{i}}=\frac{\sum_{t=1}^{T} p\left(i \mid x_{t}, \lambda\right) x_{t}}{\sum_{t=1}^{T} p\left(i \mid x_{t}, \lambda\right)} \\
\overline{\sum i}=\frac{\sum_{t=1}^{T} p\left(i \mid x_{t}, \lambda\right)\left(x_{t}-\mu_{i}\right)\left(x_{t}-\mu_{i}\right)^{\prime}}{\sum_{t=1}^{T} p\left(i \mid x_{t}, \lambda\right)}
\end{gathered}
$$

After serval iterations, the posteriori probability of feature vector $x_{t}$ can be calculated as follows:

$$
p\left(i \mid x_{t}, \lambda\right)=\frac{w_{i} g\left(x_{t} \mid \mu_{i}, \sum i\right)}{\sum_{i=1}^{M} w_{i} g\left(x_{t} \mid \mu_{i}, \sum i\right)}
$$

To recognize the type of structure landmark, the normalized feature vector $V_{s}$ and $V_{g}$ is used as an input of GMM. The number of Gaussian components is set to 3. The posteriori probability of each type of structure landmark can be calculated by using Equation (12). Figure 4 shows the GMM result of LL and LT landmark, in which the $X$-axis is the $V_{s}$ value, the $Y$-axis is the $V_{g}$ value and the $Z$-axis represents the posteriori probability.

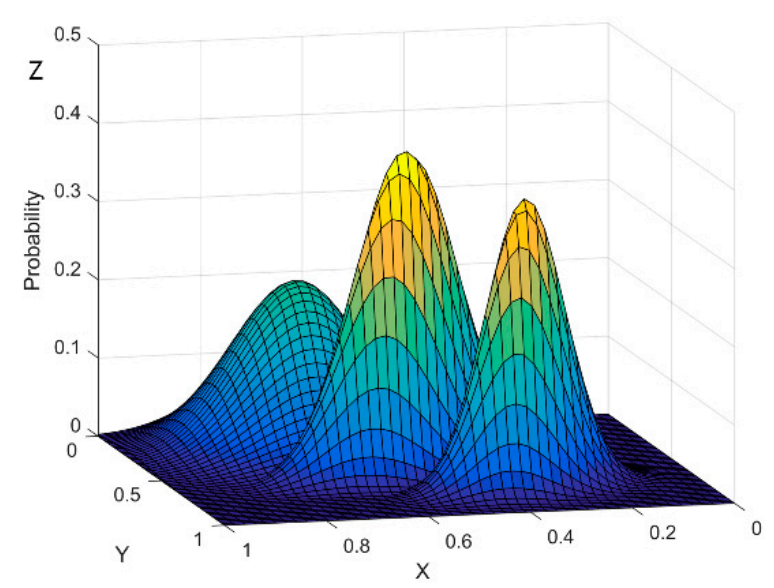

(a) the GMM of LL landmark

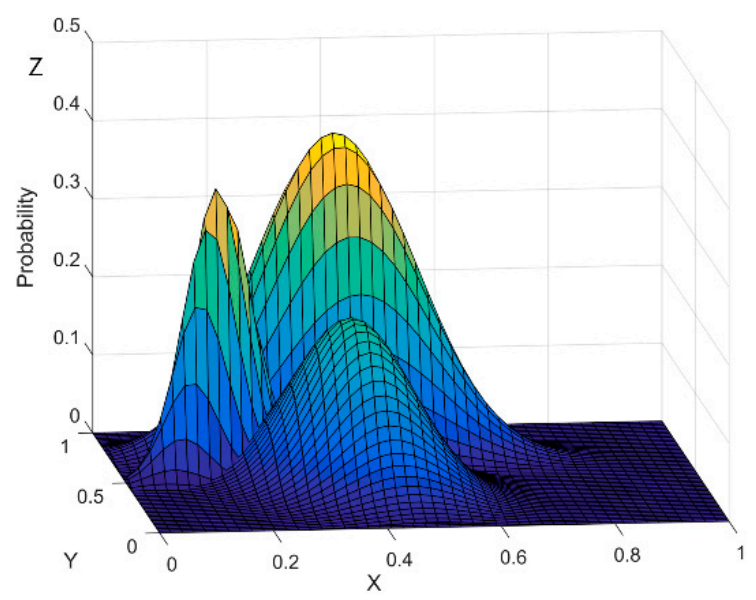

(b) the GMM of LT landmark

Figure 4. An example of GMM result of structure landmark. (a) is the LL type of landmark, (b) is the LT type of landmark

\subsubsection{Structure Landmark Recognition}

Based on the GMM-NBC model, this study uses a decision tree to identify the type of structure landmarks. When people walk in an indoor area, each encountered structure landmark reflects a specific signal pattern of inertial and visual features. Considering the randomness of pedestrian activity, this method uses inertial data to detect the activities related with turning or stopping. Later, the visual data is used to find whether the location of a walking activity happens at the location of a structure landmark. As shown in Figure 5, a detected walking activity will trigger the module of structure landmark recognition. Firstly, we separate the motion state by using turning detection and stationary detection. A peak detection algorithm [39] is implemented using gyroscope data, which can identify the orientation (left or right) and type (normal or U-turn) of a turning action. As recommend in [40], the variance of acceleration is used to detect the stationary state of pedestrian, and the threshold is set to 0.25 . The sum of rotation angle calculated from gyroscope is used to distinguish U-turn and normal turn, and the threshold is set to 135 degrees. Then, the 
variation of feature value $h_{a}$ is used to separate the type of structure landmark into two categories. If the value of $h_{a}$ is first increased and then decreased, the type of a landmark may fall in the scope of $\left\{\mathrm{FT} ; \mathrm{RT} ; \mathrm{RL} ; \mathrm{CW} ; \mathrm{WC}\right.$. If the value of $h_{a}$ is first decreased and then increased, the type of a landmark may fall in the scope of \{FT; LT; LL; CW; WC\}.

Finally, the GMM-NBC model is used to further distinguish the type of structure landmark. A special case is to distinguish EC landmark and non-structure. Due to the randomness of turning activity, pedestrian may stop walking and make a turn at a nonstructure location. We use the stationary detection and U-turn detection as a judgment condition. If the variance of acceleration is low and sum of rotation angle is higher than a threshold, EC landmark and non-structure location can be identified by using the GMM-NBC model. Another special case is that FT landmark is difficult to be accurately recognized. The reason is that when people make a right (or left) turn at a FT landmark, the visual information of scenes on the opposite side may not be collected by smartphone camera. The visual and inertial signal feature of FT landmark may be similar with RL or LL landmark. However, this potential incorrect recognition problem can be solved by the proposed landmark map matching algorithm. By matching a sequence of landmark (along a route) to the map, the influence of the incorrect landmark recognition results can be significantly reduced.

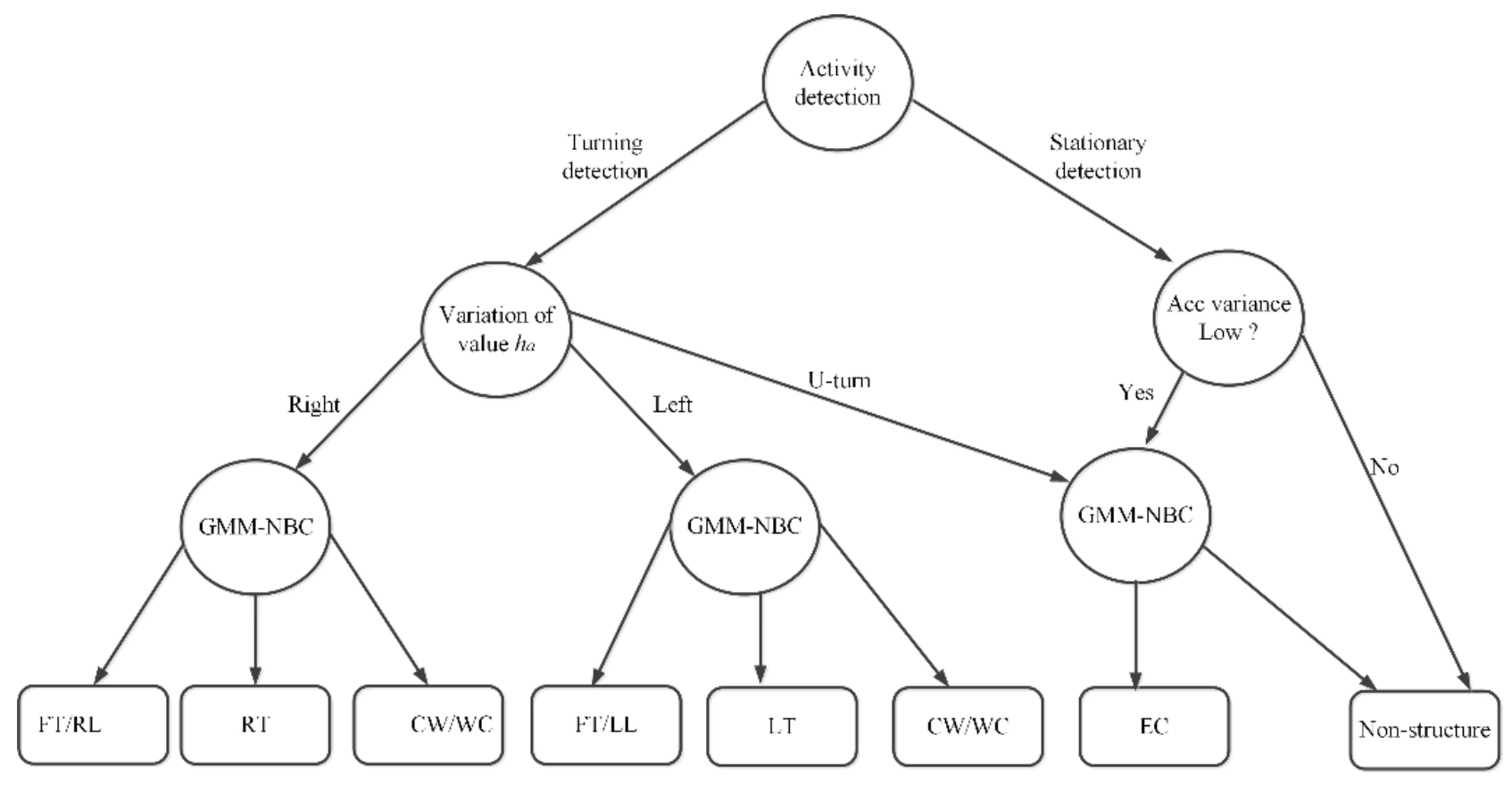

Figure 5. Decision tree for landmark recognition.

\subsection{Structure Landmark Based Indoor Map Matching}

The method proposed in Section 2.2 can be used to recognize the type of structure landmark. However, for larger indoor environments, it is possible that many structure landmarks may share the same landmark type. In order to solve this problem, a Hidden Markov model based indoor map matching algorithm is designed to uniquely identify each landmark along a signal mapping route.

The core idea of HMM is to construct the relation between the observation and the hidden state by using the conditional stochastic probability. The main parameters of HMM include: $\{\mathrm{S}, \mathrm{V}, \mathrm{A}, \mathrm{B}, \pi\}$, in which $S=\left\{S_{1}, S_{2}, S_{3}\right\}$ is the hidden state. $V=\left\{V_{1}, V_{2}, V_{3}\right\}$ is the observation. $A=\left\{a_{i j}\right\}$ is the state transition probability between state $S_{i}$ and $S_{j} . B=\left\{b_{i j}\right\}$ is the emission probability which represent the probability when the observation $V_{j}$ in the state $S_{i} . \pi=\left\{\pi_{i}\right\}$ is the initial state distribution of value $\{\mathrm{S}, \mathrm{V}, \mathrm{A}, \mathrm{B}\}$. 
In this study, we define structure landmark as the hidden state of HMM. The type of landmark, e.g., FT landmark, is defined as the observation, which can be recognized by using the method proposed in Section 2.2. The value of state transition probability $a_{i j}$ can be calculated based on the spatial adjacency relation among landmarks. The emission probability $b_{i j}$ of hidden state $S_{i}$ is defined according to the confusion matrix of landmark recognition. To increase the practicability of the proposed method, the initial location of each route is assumed to be unknown. The initial state of distribution is uniform for all hidden state. The sum of transition probability and emission probability of each hidden state equals 1. Figure 6 shows an example of calculating state transition probability and emission probability. Because landmark $S_{2}$ is directly adjacent to $S_{1}, S_{3}$ and $S_{4}$, the transition probability of $a_{21}, a_{23}, a_{24}$ are defined as $1 / 3$. For FT landmark (state $S_{2}$ ), different walking direction may lead to different identification result. Considering the existing of incorrect recognition results in confusion matrix, the emission probability of $S_{2}$ is defined as:

$$
b_{2 L T}=b_{2 R T}=b_{2 L L}=b_{2 R L}=b_{2 F T}=1 / 5
$$

where $b_{2 L T}, b_{2 R T}, b_{2 L L}, b_{2 R L}, b_{2 F T}$ represent the probability that $S_{2}$ being recognized as LT, RT, LL, RL and FT landmark.

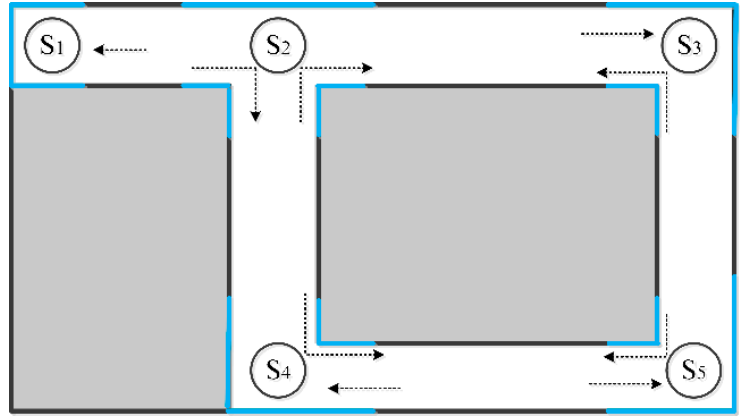

Figure 6. An example of calculating state transition probability and emission probability.

The aim of the map matching method is to find the absolute location of detected landmarks along a signal mapping route. After constructing the parameters of HMM, a Viterbi algorithm [41] is used to search for the most probable sequence of hidden state. For most indoor environments, there are many structure landmarks that share the same landmark type. The result of traditional Viterbi algorithm converges slowly and may provide more than one candidate location. In this study, we use the spatial adjacency of landmarks and the absolute heading angle information to improve the convergence speed of Viterbi algorithm. The spatial adjacency relationship of two landmarks means they can be straightly accessed by each other without turning action. Absolute heading angle information can be obtained from smartphone sensor. The main steps of this map matching algorithm are shown as below:

(1) Initialization: For the first detected and recognized landmark, all landmarks in the map will be treated as its candidate locations if they are within the same landmark type. We define $\delta_{t}(i)$ as the probability along a candidate path, at time $\mathbf{t}$, which accounts for the observation $V_{t}$ in state $S_{i}$.

(2) Induction: For subsequently recognized landmarks, calculating the probability $\delta_{t}(j)$ and $\varphi_{t}(j)$ according to the transition probability $a_{i j}$ and emission probability $b_{i j}$.

$$
\begin{gathered}
\delta_{t}(j)=\left[\max _{i=1,2, \ldots N}\left(\delta_{t-1}(i) a_{i j}\right)\right] \cdot b_{j}\left(V_{t}\right) \\
\varphi_{t}(j)=\operatorname{argmax}\left(\delta_{t-1}(i) a_{i j}\right)
\end{gathered}
$$


where $\varphi_{t}(j)$ is the most probable location of observation $V_{t}$. If the value of $\varphi_{t}(j)$ equals 0 , the path will not be used as a candidate.

(3) Backtracking. When the last structure landmark has been detected, the algorithm can estimate the state of the observation and backtrack the state array based on the array of $\varphi_{t}(j)$. The path with the highest value is selected as the result. If the number of candidate path is higher than 1 , the heading angle along the route can be used to eliminate false matches.

\subsection{Radio Map Construction}

After landmark recognition and map matching, the location of a signal mapping route can be estimated. Later, sampling point from the route can be used to construct radio map for indoor localization. The location of each sampling point can be estimated by a pedestrian dead reckoning (PDR) algorithm using inertial data. A Low-pass Butter-worth filtering and peak detection algorithm [42] is used to detect the steps from a recovered route. A frequency-based mode [43] is implemented to estimate step length. The result of PDR can be described as follows:

$$
\left(\begin{array}{l}
x_{k} \\
y_{k}
\end{array}\right)=\left(\begin{array}{c}
x_{k-1}+D_{k} \cdot \sin \theta_{k} \\
y_{k-1}+D_{k} \cdot \cos \theta_{k}
\end{array}\right)
$$

where $\left(x_{k}, y_{k}\right)$ is the coordinate of sampling point $P_{k}, D_{k}$ and $\theta_{k}$ are the step length and heading direction, respectively.

Each sample point from a mapping route is associated with Wi-Fi receive strength signal (RSS) data, which can be used to construct Wi-Fi fingerprints database (radio map). The attribute of sampling points for radio map construction is shown in Table 1 . For each sample point, the coordinates are calculated through proposed map matching method and PDR algorithm, the collected RSS data include the RSS value and its AP information. To construct a radio map, an indoor space is divided into regular grids. If a trajectory passes through a grid, the sampling points from the trajectory are extracted to generate the corresponding Wi-Fi fingerprint. As shown in Figure 7, if $\mathrm{m}$ sampling points (maybe from different trajectories) are within the spatial extent of a grid, the RSS value of AP i in the grid can be calculated as:

$$
\operatorname{rss}_{i}=\frac{1}{m} \sum_{k \in G} r s s_{i}^{k}
$$

where $\operatorname{rss}_{i}$ is the value of AP i in the grid, G is the set of AP, rss ${ }_{i}^{k}$ is the $\operatorname{rss}_{i}$ of the k-th sampling point. If one grid does not have any sampling point in its spatial extent, the value of RSS should be calculated by using the RSS from its neighbor grids:

$$
\operatorname{rss}_{i}=\frac{\sum_{j} w\left(d_{j}\right) \cdot R S S\{\}}{\sum_{j} w\left(d_{j}\right)}
$$

where $R S S\{\}$ is the neighborhood grids of the current grid, $\mathrm{j}$ is the index of a grid, $w\left(d_{j}\right)$ is the weight function which inverse distance $d_{j}$.

Table 1. The attributes of sampling points for radio map construction.

\begin{tabular}{cccc}
\hline Point ID & Trajectory ID & Coordinates & RSS \\
\hline P_1 & $\mathrm{Tr}_{-} 1$ & $(\mathrm{X} 1, \mathrm{Y} 1)$ & $\left.\left\{\left(r s s_{1}, a p_{1}\right),\left(r s s_{2}, a p_{2}\right) \ldots\right\}\right\}$ \\
$\mathrm{P} \_2$ & $\mathrm{Tr} \_2$ & $(\mathrm{X} 2, \mathrm{Y} 2)$ & $\left.\left\{\left(r s s_{1}, a p_{1}\right),\left(r s s_{2}, a p_{2}\right) \ldots\right\}\right\}$ \\
$\ldots$ & $\ldots$ & $\ldots$ & $\ldots$ \\
P_n & $\mathrm{Tr} \_\mathrm{n}$ & $(\mathrm{Xn}, \mathrm{Yn})$ & $\left.\left\{\left(r s s_{1}, a p_{1}\right),\left(r s s_{2}, a p_{2}\right) \ldots\right\}\right\}$ \\
\hline
\end{tabular}




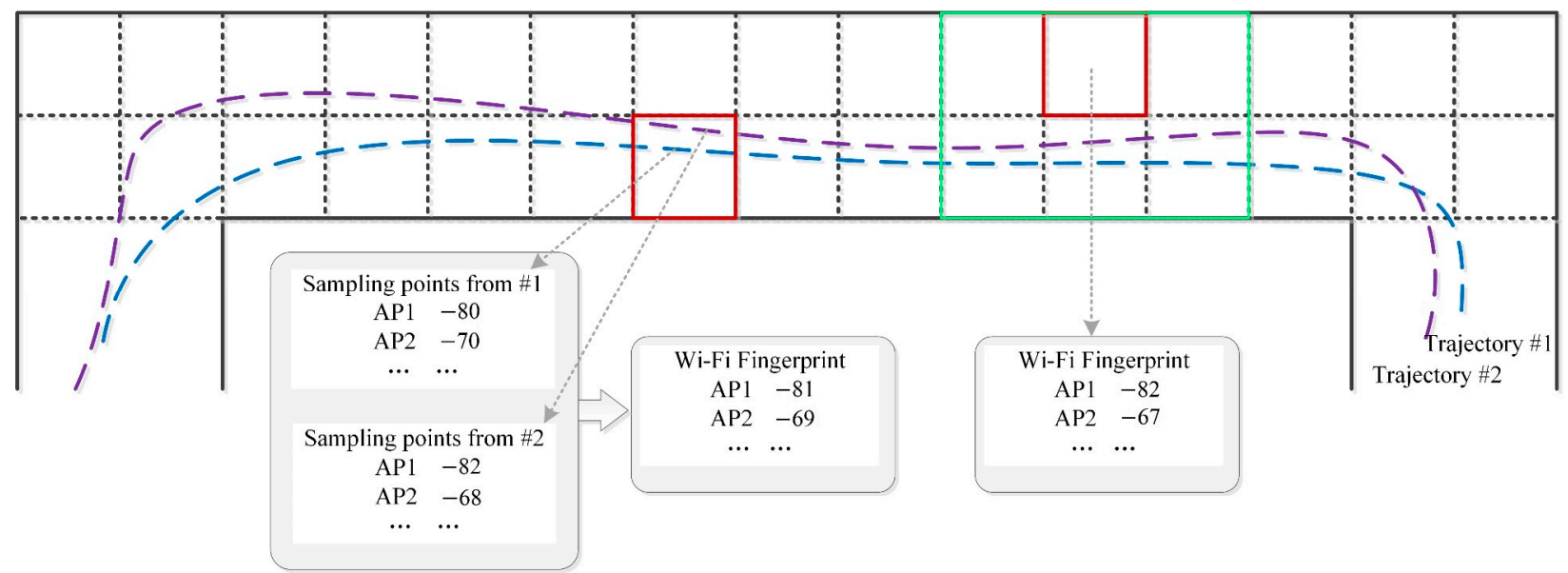

Figure 7. An example of generating Wi-Fi fingerprint.

\section{Experimental Results}

\subsection{Overview}

The performance of the proposed indoor signal mapping method is evaluated by several experiments conducted in an office building. The experimental area covers a $52.5 \mathrm{~m} \times 52.5 \mathrm{~m}$ floor plan. As shown in Figure 8a, there are total 19 structure landmarks (L1-L19) which cover 8 landmark types as defined in Section 2.1. Two Android version smartphone (a Galaxy Note and a Nexus $S$ ) are used to collect sensor data, including video frames (30FPS), inertial data (100 HZ) and Wi-Fi RSS data (150 HZ).

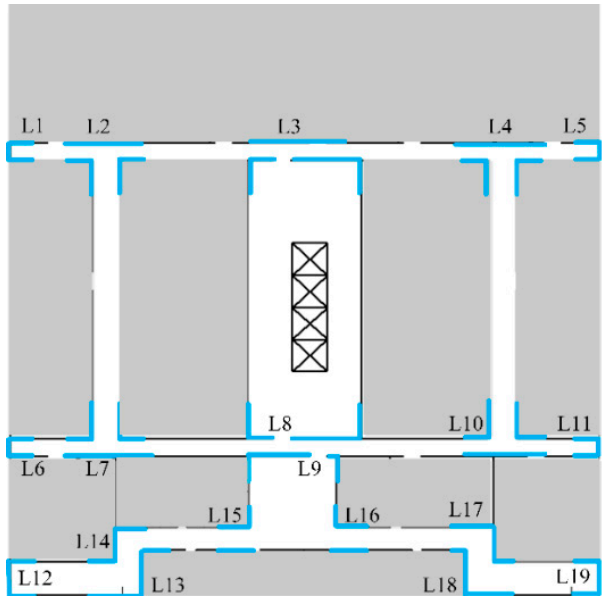

(a)

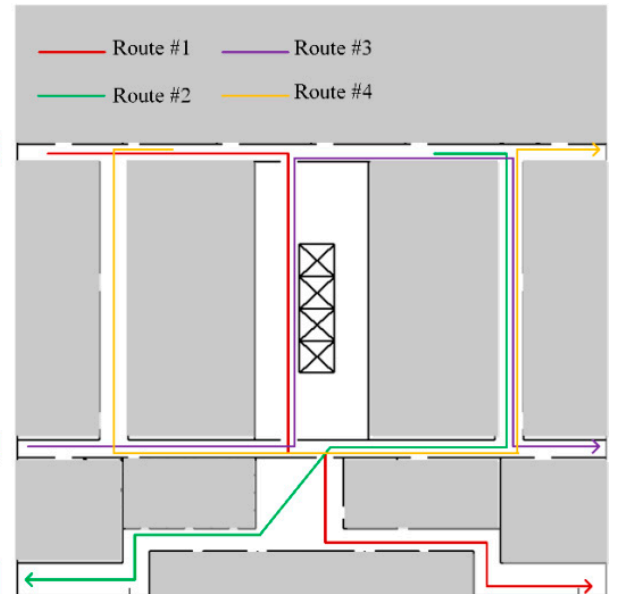

(b)

Figure 8. Experimental environment. (a) shows the number of structure landmarks, (b) shows the walking trajectory of collecting data

To test the accuracy of landmark recognition method, twenty sets of sample data (for each landmark type) were collected in different indoor spaces. The accuracy of landmark recognition is calculated as:

$$
\text { Accuracy }=M / N \cdot 100 \%
$$

where $\mathrm{M}$ is the number of correctly recognized structure landmarks, $\mathrm{N}$ is the total number of landmarks. The confusion matrix of landmark recognition results are summarized in Table 2. The accuracy of most of the landmark type is higher than $90 \%$. Especially LL, RL and EC, where the recognition accuracy is $100 \%$. For FT landmark, the recognition accuracy is around $50 \%$. However, the negative influence of incorrectly recognized landmarks can be reduced by the map matching method. 
Table 2. The recognition result of landmark type.

\begin{tabular}{ccccccccc}
\hline & FT & LT & RT & LL & RL & EC & CW & WC \\
\hline FT & $50 \%$ & 0 & 0 & $25 \%$ & $25 \%$ & 0 & 0 & 0 \\
LT & 0 & $95 \%$ & 0 & $5 \%$ & 0 & 0 & 0 & 0 \\
RT & 0 & 0 & $95 \%$ & 0 & $5 \%$ & 0 & 0 & 0 \\
LL & 0 & 0 & 0 & $100 \%$ & 0 & 0 & 0 & 0 \\
RL & 0 & 0 & 0 & 0 & $100 \%$ & 0 & 0 & 0 \\
EC & 0 & 0 & 0 & 0 & 0 & $100 \%$ & 0 & 0 \\
CW & 0 & $5 \%$ & $5 \%$ & 0 & 0 & 0 & $90 \%$ & 0 \\
WC & 0 & 0 & 0 & $5 \%$ & $5 \%$ & 0 & 0 & $90 \%$ \\
\hline
\end{tabular}

\subsection{Performance of Indoor Map Matching}

To evaluate the performance of the structure landmark-based map matching method, four participants were required to collect experiment data along four designed routes (shown in Figure $8 b$ ). Each route was repeated 10 times. The initial location of each route was assumed to be unknown. The proposed map matching method was used to estimate the location of the four routes. The location of identified structure landmark is used as metric for evaluating the accuracy of landmark map matching. To obtain the quantitative assessment of the method, some markers with known coordinates were set along each route to collect ground truth data. The location error can be calculated as follows:

$$
\text { Error }_{i}=\left|P_{e i}-P_{g i}\right|
$$

where Error ${ }_{i}$ is the location error of the $\mathrm{i}$-th ground-truth point, $P_{e i}$ is the estimated location of estimated point, $P_{g i}$ is the ground-truth point.

The result of structure landmark-based map matching is shown in Table 3. The matching accuracy of four routes is higher than $90 \%$ and the average location error is less than $1.6 \mathrm{~m}$. Generally, map matching accuracy is higher than structure identification accuracy. For Route \#1, although two times of incorrect landmark recognition happened at L16, this method can provide a precise map matching result. Similarly, for Route \#2, although landmark L10 and L15 are incorrectly recognized as RL and LL respectively, the landmark matching accuracy reaches $98 \%$. In some cases, the error of map matching comes from the incorrect recognition of landmark type. For Route \#3, an incorrect recognition happens at L8, which causes the incorrect map matching of the following several landmarks. The relatively low recognition accuracy of Route \#4 is due to the incorrect recognition of a FT landmark. However, although the landmark recognition accuracy for all routes is about $84 \%$, the map mapping accuracy reaches $96 \%$. It indicates that the proposed map mapping method can significantly reduce the negative influence of incorrect landmark recognition result.

Table 3. Map matching result of four routes.

\begin{tabular}{cccccc}
\hline Route & $\begin{array}{c}\text { Route } \\
\text { Length }(\mathbf{m})\end{array}$ & $\begin{array}{c}\text { Detected } \\
\text { Landmark }\end{array}$ & $\begin{array}{c}\text { Recognition } \\
\text { Accuracy }\end{array}$ & $\begin{array}{c}\text { Matching } \\
\text { Accuracy }\end{array}$ & $\begin{array}{c}\text { Average } \\
\text { Error (m) }\end{array}$ \\
\hline$\# 1$ & 86.8 & 70 & $97 \%$ & $97 \%$ & 1.07 \\
$\# 2$ & 87.1 & 70 & $88 \%$ & $98 \%$ & 1.25 \\
$\# 3$ & 102.1 & 50 & $82 \%$ & $90 \%$ & 1.53 \\
$\# 4$ & 102.4 & 50 & $60 \%$ & $100 \%$ & 0.98 \\
\hline
\end{tabular}

Compare with activity-based map matching method [39,40], the offline localization error of structure landmark based matching is smaller. Figure 9 shows the offline localization results of the four routes using activity based and structure-based map matching methods respectively. The average error of activity-based map matching $(2.2 \mathrm{~m})$ is obviously higher than structure based map matching $(1.2 \mathrm{~m})$. The results indicate that by integrating both visual and inertial features, structure landmark can serve as a more robustness spatial 
anchor point for map matching than activity landmark. The visual details of the four estimated routes are shown in Figure 10.

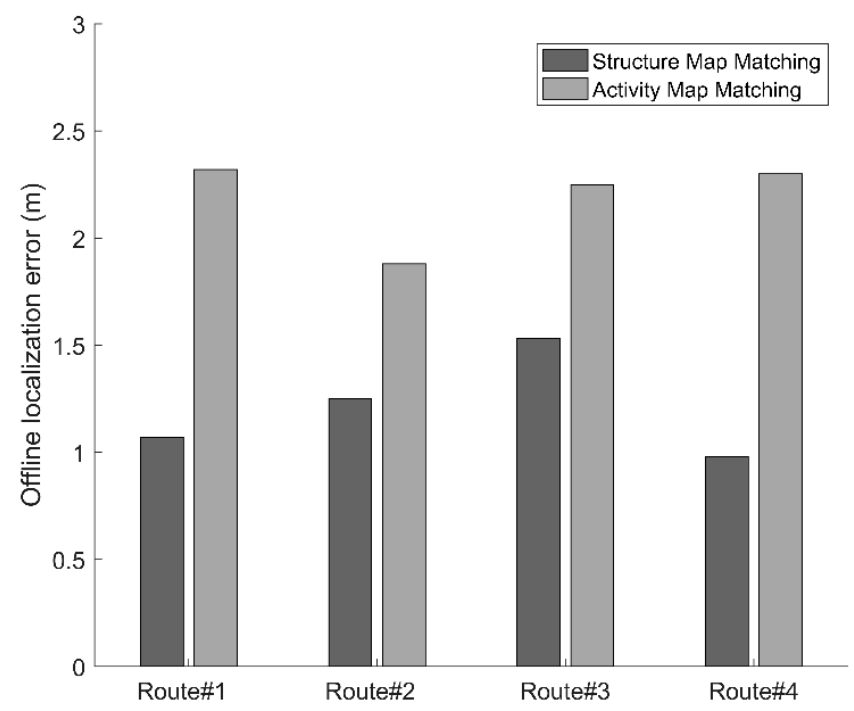

Figure 9. Performance of offline localization.

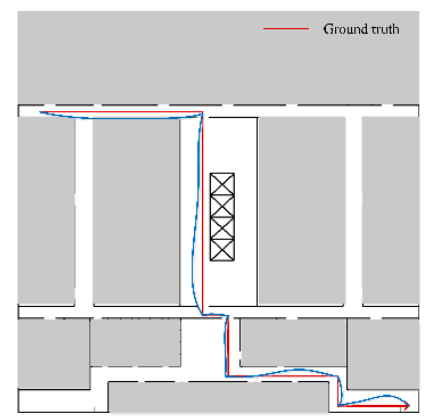

Route\#1

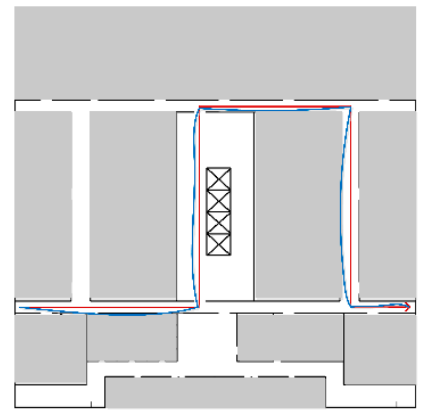

Route \#3

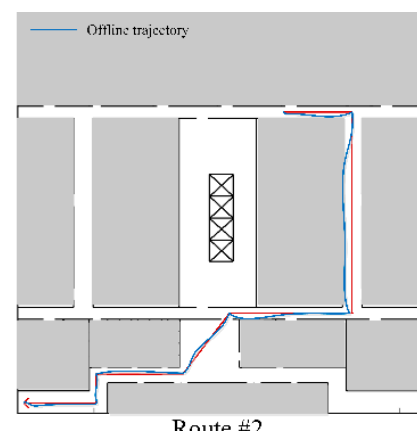

Route \#2

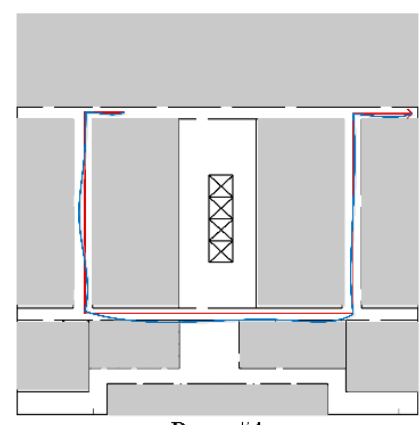

Route \#4

Figure 10. Recovered results of four signal mapping routes.

\subsection{Performance of Radio Map Construction}

To test the performance of the proposed signal mapping method, we used the sampling points from the four routes to construct a radio map for the study area, then conducted an indoor localization experiment using the constructed radio map. First, the study area was partitioned into a $1 \mathrm{~m} \times 1 \mathrm{~m}$ mesh grid. The sampling points from the routes were extracted to a radio map by using the method described in Section 2.4. Figure 11 shows the RSS distribution of two APs in the constructed radio map. The RSS of APs in the radio map ranges from $-55 \mathrm{dBm}$ to $-88 \mathrm{dBm}$. 

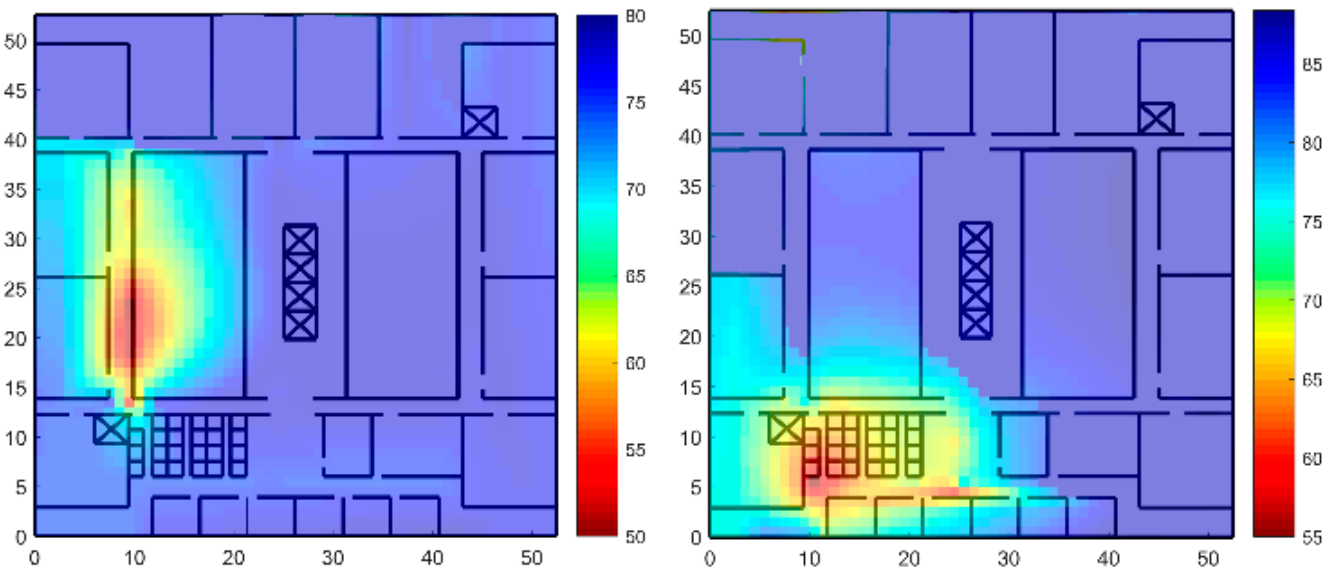

Figure 11. The RSS distribution of two APs.

To evaluate the quality of the constructed radio map, two online localization experiments were implemented by using the weighted K-nearest neighbor (KNN) approach [44] and Deep neural network (DNN) approach [45], respectively. The number of used mapping routes (for constructing radio map) was taken as a variable for the two experiments.

Figure 12 shows the localization performance of two methods under three different conditions (T1, T2 and T3). T1 represents that the radio map was constructed using only one mapping route. $\mathrm{T} 2$ and $\mathrm{T} 3$ represent the radio map was constructed using five routes and ten routes, respectively. The reason for using the three conditions is to evaluate the influence of mapping route number on the quality of constructed radio map. The average error of T1 by using KNN and DNN are $3.7 \mathrm{~m}$ and $4.0 \mathrm{~m}$, respectively. The error of KNN is smaller than DNN. It means that in the case of less route data, the performance of deterministic method is better than learning based method. It has been observed that with the increase in used mapping routes (from T1 to T2), there is significant improvement in the quality of constructed radio map. The average location error of the two methods is $1.7 \mathrm{~m}$ and $1.6 \mathrm{~m}$, respectively. With further increase in used routes (from T2 to T3), the average location error of both KNN and DNN reached to $1.6 \mathrm{~m}$ respectively. Compared with T2, the error of $\mathrm{T} 3$ decreases. These results indicate that the increase in mapping routes can help to increase the quality of constructed radio map. However, when there are enough mapping route data, the further increase of mapping route will not continuously improve the quality of radio map. The constructed radio map can provide a reliable localization results in indoor spaces.

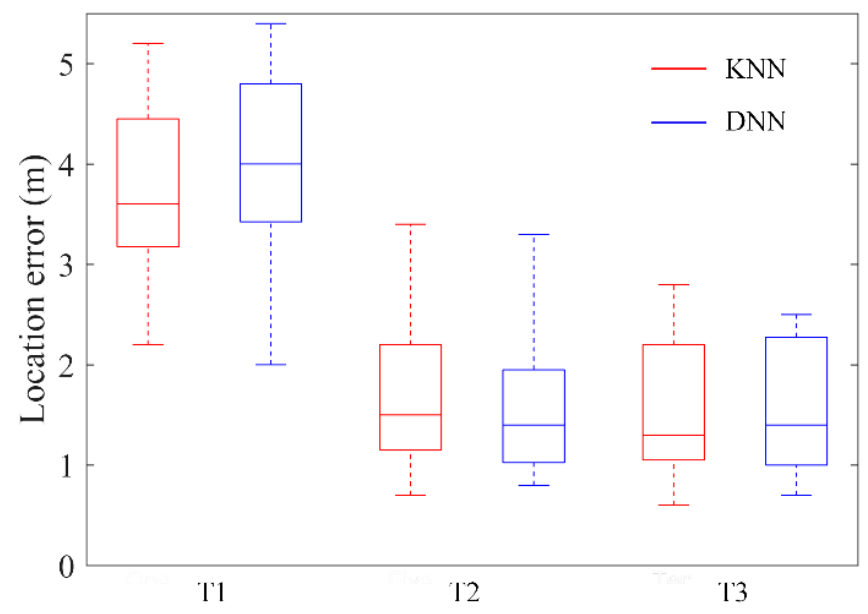

Figure 12. Localization result of two methods using three type of radio maps. T1 is the location error using one mapping route, $\mathrm{T} 2$ and $\mathrm{T} 3$ are the location error using five and ten routes respectively. 


\subsection{Sustainability Evaluation of the Proposed Method}

To evaluate the sustainability of this method, three different radio map construction methods, including a site survey method [4], an activity landmark-based mapping method [39] and the proposed method, were implemented in a new study area (in a hospital). As shown in Figure 13a, this area covers a $62 \mathrm{~m} \times 60 \mathrm{~m}$ floor plan. It totally contains 20 structure landmarks. Multisource data from smartphone is collected along three trajectories and each trajectory repeated 5 times. To implement the site survey method, the study area is divided into $2 \mathrm{~m} \times 2 \mathrm{~m}$ grids. A smartphone is used to collect Wi-Fi data at the center of each grid for about $50 \mathrm{~s}$. Figure 13b shows the localization results by using the three methods. The average localization error of the site survey method, the activity landmark-based method and the proposed method is $2.3 \mathrm{~m}, 4.2 \mathrm{~m}$ and $3.6 \mathrm{~m}$. The location accuracy of the proposed structure landmark-based method is higher than the activity landmark-based method. Moreover, the time cost of the proposed method (about $15 \mathrm{~min}$ ) is obviously lower than the site survey method (about $2 \mathrm{~h}$ ). It shows that although the localization error of the proposed method is higher than the traditional site survey method, it can achieve a relatively high accuracy and greatly reduce the time cost and human labor required for fingerprint collection.

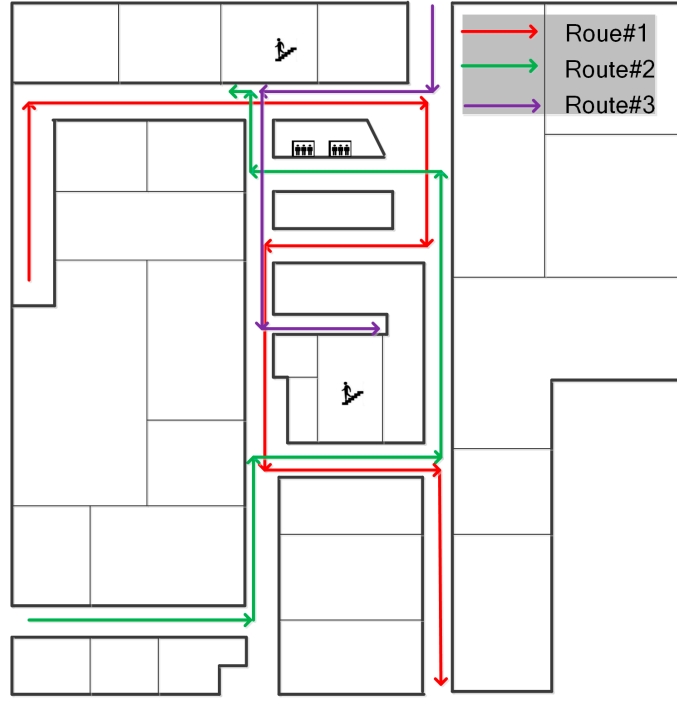

(a)

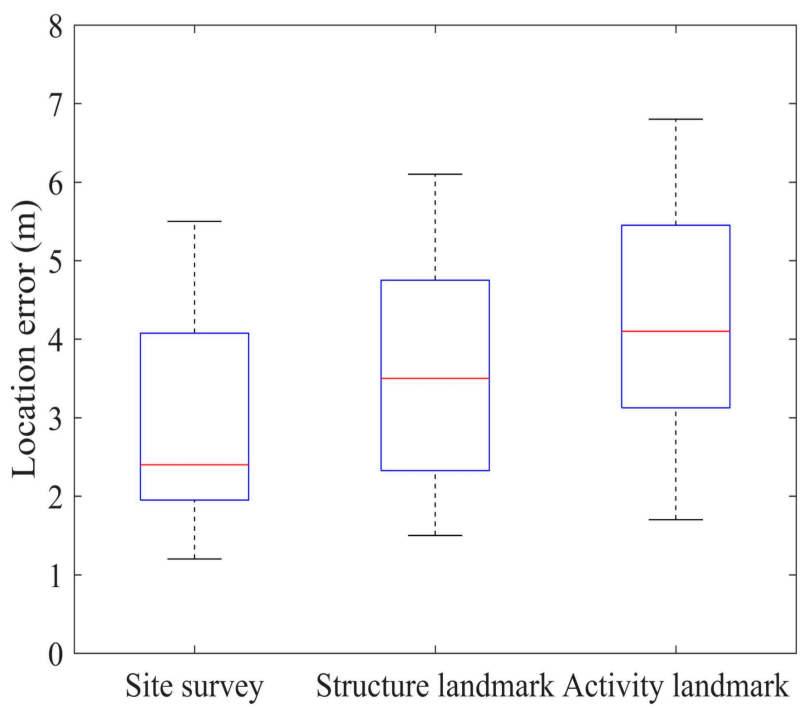

(b)

Figure 13. Localization result of three different methods at a hospital. (a) is the hospital area, (b) is the localization results

\section{Conclusions}

This paper proposes a structure landmark map matching based indoor radio map construction approach. 8 structure landmark type is formally defined. A GMM-NBC model is designed to recognize the type of structure landmarks in indoor space. A structure landmark-based map matching and radio map construction modules are also developed for constructing indoor radio map. The performance of the proposed approach has been evaluated by conducting several experiments. The result showed that this method could accurately estimate the spatial location of signal mapping route without knowing its initial location. The sampling points from mapping route can be extracted to construct a radio map for indoor localization. By using a constructed radio map, two localization algorithms (KNN and DNN) can achieve a localization accuracy of $1.6 \mathrm{~m}$, which demonstrates that the constructed radio map has a reliable quality for indoor localization. Furthermore, the proposed approach can reduce the workload and time cost for fingerprints collecting and radio map construction, which is practical for the deployment of indoor fingerprintingbased localization systems and applications. 
Author Contributions: Conceptualization, T.L. and X.Z.; methodology, T.L.; software, X.Z.; validation, T.L., X.Z. and H.Z.; formal analysis, T.L.; investigation, T.L.; resources, N.T. and Z.F.; data curation, T.L.; writing—original draft preparation, T.L.; writing—review and editing, T.L., X.Z. and N.T.; visualization, X.Z.; supervision, H.Z.; project administration, Z.F.; funding acquisition, T.L. and X.Z. All authors have read and agreed to the published version of the manuscript.

Funding: This work was supported by the National Natural Science Foundation of China (Grant No. 41801376, 42071434, 41771473); China Postdoctoral Science Foundation (No. 2020M682293); Natural Science Foundation of Guangdong Province (2018A030313289), Shenzhen Scientific Research and Development Funding Program (JCYJ20170818144544900, JCYJ20180305125033478), Open Research Fund of state key laboratory of information engineering in surveying, mapping and remote sensing, Wuhan University (18S03), Key Research Projects of Henan Higher Education Institutions (19A420004,19A420002).

Institutional Review Board Statement: Not applicable.

Informed Consent Statement: Not applicable.

Data Availability Statement: Not applicable.

Conflicts of Interest: The authors declare no conflict of interest.

\section{References}

1. Park, J.H.; Chao, H.-C. Advanced IT-Based Future Sustainable Computing. Sustainability 2017, 9, 757.

2. Sung, Y. RSSI-Based Distance Estimation Framework Using a Kalman Filter for Sustainable Indoor Computing Environments. Sustainability 2016, 8, 1136.

3. Cai, X.; Gao, Y.; Li, M.; Song, W. Infrared Human Posture Recognition Method for Monitoring in Smart Homes Based on Hidden Markov Model. Sustainability 2016, 8, 892.

4. Bahl, P.; Padmanabhan, V.N. RADAR: An in-building RF-based user location and tracking system. In Proceedings of the Nineteenth Annual Joint Conference of the IEEE Computer and Communications Societies (INFOCOM), Tel Aviv, Israel, 26-30 March 2000; Volume 2, pp. 775-784.

5. Namkyoung, L.; Sumin, A.; Dongsoo, H. AMID: Accurate Magnetic Indoor Localization Using Deep Learning. Sensors 2018, 18, 1598.

6. Pengzhan, C.; Ye, K.; Xiaoyue, C. A UWB/Improved PDR Integration Algorithm Applied to Dynamic Indoor Positioning for Pedestrians. Sensors 2017, 17, 2065.

7. Shao, W.; Luo, H.; Zhao, F.; Ma, I.; Zhao, Z.; Crivello, A. Indoor positioning based on fingerprint-image and deep learning. IEEE Access 2018, 6, 74699-74712.

8. Ye, F.; Chen, R.; Guo, G.; Peng, X.; Liu, Z.; Huang, L. A Low-Cost Single-Anchor Solution for Indoor Positioning Using BLE and Inertial Sensor Data. IEEE Access 2019, 7, 162439-162453.

9. Lou, W.; Wu, J. Double-covered broadcast (dcb): A simple reliable broadcast algorithm in manets. In Proceedings of the IEEE INFOCOM, Hong Kong, China, 7-11 March 2004.

10. Stann, F.; Heidemann, J.; Shroff, R.; Murtaza, M.Z. Rbp: Robust broadcast propagation in wireless networks. In Proceedings of the 4th International Conference on Embedded Networked Sensor Systems (Sensys), Boulder, CO, USA, 31 October-3 November 2006.

11. Wang, W.; Liu, X.; Yao, Y.; Pan, Y.; Chi, Z.; Zhu, T. CRF: Coexistent Routing and Flooding using WiFi Packets in Heterogeneous IoT Networks. In Proceedings of the IEEE Conference on Computer Communications, Paris, France, 29 April-2 May 2019 ; pp. 19-27.

12. Davaslioglu, K.; Soltani, S.; Erpek, T.; Sagduyu, Y. DeepWiFi: Cognitive WiFi with Deep Learning. IEEE Trans. Mob. Comput. 2019, 99, 429-444.

13. Gilani, S.S.A.; Qayyum, A.; Rais, R.N.B.; Bano, M. SDNMesh: An SDN based Routing Architecture for Wireless Mesh Networks. IEEE Access 2020, 8, 136769-136781.

14. Han, S.; Gong, Z.; Meng, W.; Li, C. An indoor radio propagation model considering angles for WLAN infrastructures. Wireless Commun. Mob. Comput. 2015, 15, 2038-2048.

15. Sun, Y.; He, Y.; Meng, W.; Zhang, X. Voronoi diagram and crowdsourcing-based radio map interpolation for GRNN fingerprinting localization using WLAN. Sensors 2018, 18, 3579.

16. Sorour, S.; Lostanlen, Y.; Valaee, S.; Majeed, K. Joint Indoor Localization and Radio Map Construction with Limited Deployment Load. IEEE Trans.Mob. Comput. 2015, 14, 1031-1043.

17. Bolliger, P. Redpin-adaptive, zero-configuration indoor localization through user collaboration. In Proceedings of the first ACM International Workshop on Mobile Entity Localization and Tracking in GPS-less Environments, San Francisco, CA, USA, 19 September 2008; pp. 55-60.

18. Wang, H.; Sen, S.; Mariakakis, A.; Elgohary, A.; Farid, M.; Youssef, M.; Roy Choudhury, R. Video: Unsupervised indoor localization (UnLoc): Beyond the prototype. In Proceedings of the 12th Annual International Conference on Mobile Systems, Applications, and Services, Bretton Woods, NH, USA, 16-19 June 2014; p. 393. 
19. Rai, A.; Chintalapudi, K.K.; Padmanabhan, V.N.; Sen, R. Zee: Zero-effort crowdsourcing for indoor localization. In Proceedings of the 18th Annual International Conference on Mobile Computing and Networking, Istanbul, Turkey, 22-26 August 2012; pp. 293-304.

20. Hsieh, C.H.; Chen, J.Y.; Nien, B.H. Deep Learning-Based Indoor Localization Using Received Signal Strength and Channel State Information. IEEE Access 2019, 7, 33256-33267.

21. Koike-Akino, T.; Wang, P.; Pajovic, M.; Sun, H.; Orlik, P.V. Fingerprinting-Based Indoor Localization with Commercial MMWave WiFi: A Deep Learning Approach. IEEE Access 2020, 8, 84879-84892.

22. Abbas, M.; Elhamshary, M.; Rizk, H.; Torki, M.; Youssef, M. WiDeep: WiFi-based Accurate and Robust Indoor Localization System using Deep Learning. In Proceedings of the IEEE International Conference on Pervasive Computing and Communications (Percom), Kyoto, Japan, 11-15 March 2019.

23. Khatab, Z.E.; Hajihoseini, A.; Ghorashi, S.A. A Fingerprint Method for Indoor Localization Using Autoencoder Based Deep Extreme Learning Machine. IEEE Sens. Lett. 2018, 2, 1-4.

24. Wang, R.; Li, Z.; Luo, H.; Zhao, F.; Shao, W.; Wang, Q. A Robust Wi-Fi Fingerprint Positioning Algorithm Using Stacked Denoising Autoencoder and Multi-Layer Perceptron. Remote Sens. 2019, 11, 1293.

25. Wu, C.; Yang, Z.; Liu, Y.; Xi, W. WILL: Wireless indoor localization without site survey. IEEE Trans. Parallel Distrib. Syst. 2012, 24, 839-848.

26. Wu, C.; Yang, Z.; Liu, Y. Smartphones Based Crowdsourcing for Indoor Localization. IEEE Trans. Mob. Comput. 2015, 14, 444-457.

27. Yu, W.; Zhang, J.; Xu, J.; Xu, Y. An accurate indoor map matching algorithm based on activity detection and crowdsourced Wi-Fi. Sci. China Tech. Sci. 2019, 62, 1492-1501.

28. Zhou, B.; Li, Q.; Mao, Q.; Tu, W. A robust crowdsourcing-based indoor localization system. Sensors 2017, 17,864 .

29. Cao, J.; Zhao, Y.; Lai, X.; Ong, M.E.H.; Yin, C.; Koh, Z.X.; Liu, N. Landmark recognition with sparse representation classification and extreme learning machine. J. Franklin Inst. 2015, 352, 4528-4545.

30. da Cunha, K.B.; Maggi, L.; Teichrieb, V.; Lima, J.P.; Quintino, J.P.; da Silva, F.Q.; Santos, A.L.M.; Pinho, H. Patch PlaNet: Landmark Recognition with Patch Classification Using Convolutional Neural Networks. In Proceedings of the 31st SIBGRAPI Conference on Graphics, Patterns and Images (SIBGRAPI), Parana, Brazil, 29 October-1 November 2018.

31. Federico, M.; Tomaso, F.; Andrea, P. Efficient nearest neighbors search for large-scale landmark recognition. In International Symposium on Visual Computing; Springer: Cham, Switzerland, 2018; pp. 541-551.

32. Chua, A.V.S.; Cortez, J.; Cuasay, K.S.A.; Diaz, J.P.G.; Rufo, R.A.; Tolentino, R.E. Navigation by Landmark Recognition Using Camera $360^{\circ}$. In Proceedings of the 4 th International Conference on Trends in Electronics and Informatics (ICOEI), Tirunelveli, India, 15-17 June 2020; pp. 30-36.

33. Sun, G.Q.; Zhao, J.L.; Zhu, C.D. A Novel Approach for Landmark Recognition via Crowdsourced Trajectory. In Proceedings of the 3rd International Conference on Computer Science and Application Engineering (CSAE), Sanya, China, 22-24 October 2019; pp. 1-7.

34. Lee, D.C.; Hebert, M.; Kanade, T. Geometric reasoning for single image structure recovery. In Proceedings of the IEEE Conference on Computer Vision and Pattern Recognition, Miami, FL, USA, 20-25 June 2009; pp. 2136-2143.

35. Gerogiannis, D.; Nikou, C.; Likas, A. Fast and efficient vanishing point detection in indoor images. In Proceedings of the 21st International Conference on Pattern Recognition (ICPR2012), Tsukuba, Japan, 11-15 November 2012; pp. $3244-3247$.

36. Feng, C.; Deng, F.; Kamat, V.R. Semi-automatic 3d reconstruction of piecewise planar building models from single image. In Proceedings of the 10th International Conference on Construction Applications of Virtual Reality Sendai, Japan, 4-5 November 2010; pp. 309-317.

37. Park, Y.B.; Suh, I.H. Visual Recognition of Types of Corridor Segments for Mobile Robots. Adv. Rob. 2012, 26, $1915-1937$.

38. Watanabe, H.; Muramatsu, S.; Kikuchi, H. Interval calculation of EM algorithm for GMM parameter estimation. In Proceedings of the 2010 IEEE International Symposium on Circuits and Systems, Paris, France, 30 May-2 June 2010; pp. $2686-2689$.

39. Zhou, B.; Li, Q.; Mao, Q.; Tu, W.; Zhang, X.; Chen, L. ALIMC: Activity Landmark-Based Indoor Mapping via Crowdsourcing. IEEE Trans. Intell. Transp. Syst. 2015, 16, 2774-2785.

40. Zhou, B.; Li, Q.; Mao, Q.; Tu, W.; Zhang, X. Activity Sequence-Based Indoor Pedestrian Localization Using Smartphones. IEEE Trans. Hum. Mach. Syst. 2017, 45, 562-574.

41. Churbanov, A.; Winters-Hilt, S. Implementing EM and Viterbi algorithms for Hidden Markov Model in linear memory. BMC Bioinform. 2008, 9, 224.

42. Mladenov, M.; Mock, M. A step counter service for Java-enabled devices using a built-in accelerometer. In Proceedings of the 1st International Workshop on Context-Aware Middleware and Services: Affiliated with the 4th International Conference on Communication System Software and Middleware (COMSWARE 2009), Dublin, Ireland, 23 June 2009; pp. 1-5.

43. Cho, D.K.; Mun, M.; Lee, U.; Kaiser, W.J.; Gerla, M. Autogait: A mobile platform that accurately estimates the distance walked. In Proceedings of the IEEE International Conference on Pervasive Computing and Communications (PerCom), Mannheim, Germany, 29 March-2 April 2010; pp. 116-124.

44. Dai, Z.C.; Li, X.N.; Chen, Z.Z.; He, X.L. Variable-weight Indoor Fingerprinting Localization Algorithm Based on KNN Algorithm. Comput. Eng. 2019, 6, 310-314.

45. Soro, B.; Lee, C. A Wavelet Scattering Feature Extraction Approach for Deep Neural Network Based Indoor Fingerprinting Localization. Sensors 2019, 19, 1790. 